\title{
A Novel Method of Traffic Lights Adaptive Control on the Urban Road Intersections ${ }^{1}$
}

\author{
Huanjiong Zhang \\ (Hangzhou Dianzi University;Hangzhou,310018)
}

Keywords: Urban Road; Road Intersections; Traffic Lights; Adaptive Control

\begin{abstract}
: the traffic lights on urban road intersections is one of important methods to control the traffic flow, it can valuably improve the jammed traffic situation. In this paper, an adaptive traffic lights control method on urban road intersections is designed in, it can actively adjust the control time of the traffic lights based on the concrete traffic distribution in real time, so it can realize the dynamic balance between the traffic lights control time and the time that the waiting vehicle pass through the road intersections, so it can avoid taking place the jammed situation, and increase the road utilization.
\end{abstract}

\section{Introduction}

In urban areas, the roads always have many intersections with different directions. So traffic tools which pass through the road intersection must be guided, separated and control, then they can quickly, securely and orderly pass through the intersection with high availability.

The traffic lights which are installed on the intersection make key role of separating traffic tools and avoiding traffic accidents, but if the control time of the traffic tools passing or stopping in the intersection does mot match the real time which the traffic flow pass through the intersection, then some traffic accidents will take place, the most ordinary situation is that the traffic tools make long queue up on one side of the intersection because the control time which the traffic tools are allowed to pass through the intersection at this direction is too short, on the contrast, the situation is that there are no traffic tools on one side road because the control time which the vehicles are allowed to pass through the intersection at this direction is too long, and many traffic tools which are from at the other side direction of the intersection have to make queue up too, all of the above kinds of situations always lead to decrease the availability of the communication and last the driving time of the drivers and passengers, so what traffic lights are installed properly expresses the significance of the whole urban transport system.

To analyze the defects of the technology of traffic lights control, the main problem is that the traffic lights control time is static after they are installed, so the performance of the control time can not reflect the performance of the change of the traffic flow on the roads which include the intersection, furthermore this factor becomes a reason of making some traffic accidents [1-5].

In this paper, based on the analysis of the characters of the traffic lights control at the road intersection, a novel method of adaptive control is designed in, the key idea of this adaptive method is that the traffic flow on the roads that include the intersection always varies, so the control time of vehicles passing and stopping on the scene of the intersection must change too, if the control time of the traffic lights also changes according to the change of the concrete traffic flow, this is that they match each other, so the availability of the transportation can increase and the ratio of the traffic accidents can decrease.

\section{Analysis of the Characters of the traffic flow on the intersection and the Principle of the Adaptive Control}

There are two different classifications of intersection on the urban roads, one is called the three-fork intersection, the other is called the four-fork intersection. No matter what kinds of then,

${ }^{1}$ Supported by the transportation item of Taizhou section. 
the traffic tools which want to pass through the intersection must accept the control of the traffic lights at all directions, the traffic tools are permitted to pass through the intersection directly, and the same time ,another traffic tools which are at the different directions have to stop on the road waiting. In these whole processing, the passing time which is defined that the traffic tools are permitted to pass through the intersection and the stopping time which is defined the traffic tools have to stop on the roads are become the key roles, they really become the rhythm to influence the traffic situation on the intersection. The traditional processing method of traffic lights control is that the passing time and stopping time which are determined change no longer, so this control time is static, but the traffic flow on this intersection is always changing, so it is clear that the static control time is not suitable for the dynamic traffic flow. Because of this reason, some occasions, the queue of the traffic at one direction or the empty with long time at another direction appears, they become the concrete traffic accident to become the reason to decrease the transportation availability of the concrete road.

In order to increase the transportation availability of the concrete road with the intersection, the important measurement is to improve the performance of the traffic lights, especially, if the static control time which is determined when the traffic lights were installed on the intersection becomes to make the dynamic change based on the traffic flow in real time. Because the traffic flow is a random sequence, it is always changing during the whole traffic processing based on the factors of the urban road conditions and the different durations. Especially, the factor of different durations make the key role of the whole factors, in a word, the duration is different, then the traffic flow is different too, for examples, if the whole period is considered as one day, then during the duration of the peak of going on and off duty, the traffic flow appears heavy, and during the duration of deep night, it is clear the traffic flow does not become heavy, in the same way, if the whole period is considered as one month or one year, the function of the traffic flow also expresses the different performances.

Based on these regulars of the traffic flow on the intersection, a novel adaptive control method can is conceived of making the dynamic control time substitute for the static control time, that is the control time is changed based on the change of the traffic flow, the general regular is that when the waiting queue of the traffic flow at one direction is longer than the threshold which had been ascertained beforehand, then the control time which the traffic flow are permitted to pass through at this direction is to be make longer than that of the initiative control time, to let the initial time is $t$, then the new control time becomes $t+\Delta t$ ( here $\Delta t$ is called the adjusting coefficient), and the control time of empty traffic flow at one direction is longer than the threshold time which is determined initially, then the new control time becomes shorter than that of the initial control time, to let the initial control time be $t$, then the new control time becomes $t-\Delta t$, of course, the third situation is that the new control time does not change because it is not satisfied with the above different cases. The regular which includes all kinds of cases can be described as the following problem:

To let the $i^{\text {th }}$ control time adjusting interval be $t_{i}\left(\{i \mid i=0,1,2, \cdots, N\}, t_{0}\right.$ is the original value stands for the initial control time), the adjusting coefficient be $\square t(\square t>0)$, the queue length of the waiting traffic flow be $l$, the threshold value of it be $l_{\text {threshold }}$ the duration of empty queue of the traffic flow be $T$, and the threshold value of it be $T_{\text {threshold }}$, then the concrete mathematic form is

$$
t_{i+1}=\left\{\begin{array}{rl}
t_{i}+\Delta t & l \geq l_{\text {threshold }} \\
t_{i} & \text { else } \\
t_{i}-\Delta t & T \geq T_{\text {threshold }}
\end{array} \quad i=0,1, \cdots, N\right.
$$

In here, the adjusting interval $t_{\text {interval }}$ is a coefficient, which is based on the real transportation situation and the considered investigating period, in ordinary cases ,the period is considered one day, then the adjusting interval can be considered as 10 minutes or 15 minutes, and so on., the aim of this kind treatment is to make the change of the control time match the change of the traffic flow which pass through this intersection of the urban roads . 


\section{The Realization of the Novel Method of the Adaptive Control}

Based on the above analysis, what the concrete adaptive control method is realized can be concluded as the following steps:

Step one: to investigate the traffic flow at the different directions on the intersection, then to set up the initial control time to the traffic lights, so all the traffic tools that pass through this intersection must carry out faithfully this original rules.

Step two: to investigate the change of the traffic flow at different directions after the initial set of the control time, and to record the queue length of the traffic tools which were waiting for passing through the intersection and the sustained time of the empty queue on the road at the different directions of the intersection.

Step three, based on the providing coefficients which are $\square t, l_{\text {threshold }}, T_{\text {threshold }}, t_{\text {interval }}$, then to utilize form(1), the new control time is obtained, the new control time substitutes for the former control time.

Step four: to repeat step three, the new control time substitute for the former value that was recently gained, this step can last in the whole processing.

With the further analysis, though the coefficients of $\square t, l_{\text {threshold }}, T_{\text {threshold }}, t_{\text {interval }}$ are determined in prior, they also can be change based on the real situation and the special requirement. in the whole adjusting processing, $\square t$ and $t_{\text {interval }}$ can change based on the change of the real traffic flow at the different directions on the intersection, in a certain sense, $\square t$ and $t_{\text {interval }}$ can be regarded as the function of the coefficients of $l$ and $T$, the whole processing expresses the intelligent character, and it becomes the very reason that this novel control method of traffic lights is called an adaptive control method.

The structure of this adaptive method is figured in Fig1:

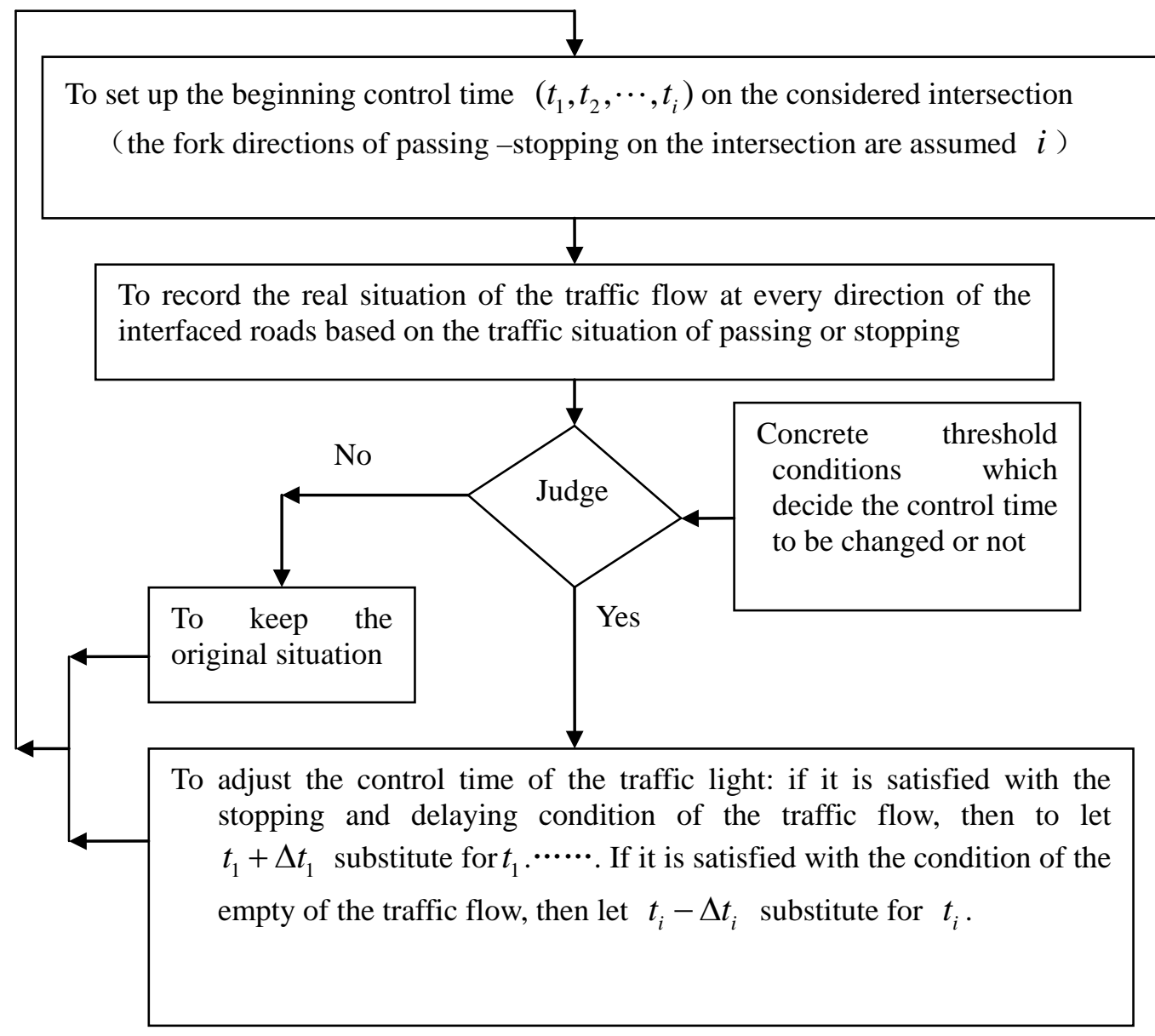

Fig1: the structure of the novel adaptive control method of traffic lights 


\section{Simulation Analysis and Conclusion}

On the theory, this adaptive method can make advanced performance in the real engineering field, in order to validate the advantages of this method is existing or not, a simple experiment is demonstrated, the result expresses that it can increase the availability of transportation, but the increasing level is influenced on the coefficients, this experiment also supports the theoretical conclusion.

This adaptive method is based on the dynamic balance between the control time of the traffic lights and the traffic flow at the different directions on the concrete urban road intersection. This new method can be regarded as the innovation of the traditional method of the traffic lights control.

\section{Reference}

[1] Huanjiong Zhang, Some Novel Innovational Technologies of Intelligent Transportation[M], the press of people transportation, Beijing 2015,8

[2] L.C. Davis, Controlling traffic flow near the transition to the synchronous flow phase,[J] Physia,A,vol.368,no.2 pp,541-550. 2006.

[3] R.Jiang, B. Jia, Q.S,Wu, the stochastic randomization effect in the on ramp system: Single lane main road and two lane main road situations[J],Journal of physics A, vol. 36, no. 47, pp11713-11723, 2003.

[4] X.M. Zhao, Z.Y. Gao, Controlling traffic jams by a feedback signal[J], European Physics Journal B, vol.43,no.4, pp565-572,2005.

[5] D. Helbing,R.Tilch, Generalized force model of traffic dynamics[J], Physical review E,vol. 58, no. 1 pp. 133-138,1998. 